\title{
Infectious Sources of Histoplasmosis and Molecular Techniques for its Identification
}

\author{
Sudip Bhandari ${ }^{1}$, Binod Rayamajhee ${ }^{2}$, Laxmi Dhungel ${ }^{1}$, Sami Poudel ${ }^{1}$, Bhagwati Gaire ${ }^{1}$, \\ Sunil Shrestha ${ }^{1}$, Niranjan Parajuli ${ }^{*}$ \\ ${ }^{1}$ Department of Biotechnology, National College, Tribhuvan University, Kathmandu, Nepal \\ ${ }^{2}$ Department of Infectious Diseases and Immunology, Kathmandu Research Institute for Biological \\ Sciences (KRIBS), Lalitpur, Nepal \\ ${ }^{3}$ Central Department of Chemistry, Tribhuvan University, Kathmandu, Nepal
}

\begin{abstract}
Histoplasmosis, a fungal infection caused by Histoplasma capsulatum (H. capsulatum), acquired from contaminated soil with droppings of chicken or birds and found to be distributed in many parts of the world. The prevalence of histoplasmosis has not well studied in Nepal. The common symptoms of acute and epidemic histoplasmosis include high fever, cough, and asthenia and weight loss. Most of the infections associated with histoplasmosis are asymptomatic. People with compromised immune systems such as HIV/AIDS (PLWHA), cancer, and organ transplant recipients are at risk of developing this disease. In this review, we have summarised the current status of histoplasmosis in Nepal and molecular techniques available for its identification. To date, the significant outbreak is not reported in Nepal, but the risk of infection for the vulnerable population cannot be undermined. Appropriate preventive measures and treatment on time can reduce the burden of this fungal disease. Further, this review is also focused on molecular identification of $H$. capsulatum. Hence, careful considerations by concerned stakeholders for national surveillance programs and the treatment of patients on time after proper diagnosis is highly recommended.
\end{abstract}

Keywords: Histoplasmosis, asymptomatic, vulnerable, treatment

*Corresponding author:

Email: nparajuli@cdctu.edu.np

\section{Introduction}

Histoplasmosis is acquired by inhalation of spores of $H$. capsulatum, which is usually found in the soil contaminated by bird droppings, which reveals the higher risk of disease in farmers, gardeners, poultry keepers, construction workers, pest control workers and in some instances travelers visiting caves and tunnels[1]. Histoplasma is a dimorphic fungus, which produces mycelial form in the soil environment and converts to the yeast form at host body temperature $\left(37^{\circ} \mathrm{C}\right)$, and it usually does not develop the symptomatic infection. So people need not concern about the infection [2]. However, for immune-compromised people, the fungus can result in severe infection. Very young children and elderly people who have a weak immune system; are more likely to get histoplasmosis disease [3].

The development of infection and the dissemination of $H$. capsulatum are initially dependent on the condition of the host body[4].
The majority of infected persons could have either no symptoms or a very less mild sickness, which is hard to recognize as the cause of histoplasmosis [5]. The clinical symptoms generally occur only in a small number (around $1 \%$ ) of the population when exposed with $\mathrm{H}$. capsulatum spores [6]. Persons who are usually immuno-compromised and are unable to develop effective cell-mediated response are likely to develop symptomatic disease during the period of acute dissemination in body [5], which includes infant child, patients with HIV/AIDS, organ transplant recipients, and those with hematologic deficiencies, and also those patients, who are on corticosteroids drugs treatment [7]. An individual is under the risk of developing symptoms even after years leaving the endemic vicinity of histoplasmosis if the man or woman was in an immuno-suppressive situation at that time [8]. Histoplasmosis is of four major types: pulmonary histoplasmosis, progressive disseminated histoplasmosis, 
cutaneous histoplasmosis, and African histoplasmosis $[9,10]$.

\section{Pathogenesis of Histoplasmosis}

The fungal infection starts after the inhalation of spores produced by the mycelial form of $H$. capsulatum and the spores deposited inside the alveoli of the lungs [11]. After some time, the spores start to germinate inside the alveoli at normal body temperature to become dimorphic form. Then pulmonary macrophages engulf that yeast form of dimorphic fungus [12]. After the engulfment, the yeasts become a parasite and multiply within the alveolar cells, then travel to hilar and mediastinal lymph nodes [13]. When yeast form fungus gets access to the blood circulation system, then disseminates more rapidly and swiftly across various organs of the body. About after two weeks of exposure, the macrophages become fully fungicidal, then the cellular immunity starts a defense against the fungal particles [14]. It leads to necrosis at the site of infection like in the lungs, liver, spleen, lymph nodes, and on bone marrow, adrenal glands, and mucocutaneous membranes, which results in progressive disseminated histoplasmosis [4] as shown in Figure 1. The progressive type of histoplasmosis is much more lethal and severe as compared to other kinds of histoplasmosis [15].

\section{Epidemiology}

Histoplasmosis is distributed worldwide, but incidence cases are often reported around the periphery of river valleys [16]. The majority of infections in humans are reported from the central United States, whereas the small number of cases are also reported from Brazil, Argentina, India, and South Africa [17]. Currently, due to an increase in the occurrence of histoplasmosis worldwide, scientists have been developing several diagnostic strategies. Histoplasmosis endemicity can be evaluated by population-based use of a histoplasmosis skin test [18]. The skin test was found to be useful when a major endemic area of histoplasmosis identified in the north-eastern and Midwestern United States [19].

Increased number of infectious diseases like histoplasmosis is being reported in areas where it was previously not thought to be prevalent, and the changing distribution of infectious diseases can become an important step to guide diagnostic workup and direct public health awareness [17]. Notably, in tuberculosisendemic regions, disseminated histoplasmosis can easily be misdiagnosed as tuberculosis because of its similar clinical symptoms and must be considered as histoplasmosis infection in patients who do not respond to empiric antitubercular therapy [20].

An estimated discern indicates that nearly 3,000 people develop kidney failure yearly in Nepal [21]. Similarly, there are additionally a high number of the population in Nepal with a diabetic condition; the 2016 Diabetes Profile has shown that $9.1 \%$ of the Nepali people are dwellings with diabetes [22]. Likewise, the cases of HIV/AIDS is also in excessive number inside the country [23]. According to National Centre for AIDS and STD Control (Nepal), there are around 31,020 HIV/AIDS sufferers in the country, and it is estimated to upward thrust at a rate of 2 patients per day. Those persons having conditions like a diabetic, organ transplant, and the person with HIV/AIDS have a weak immune system, which means that they are at high risk of developing other secondary infections like histoplasmosis during their lifetime.

According to the published reports, the geographical distribution of histoplasmosis has not been clearly understood [4]. The occurrence of histoplasmosis in Asia has not fully appreciated until recently. Malaysia is the first country in Asia, where $H$. capsulatum was isolated from soil samples [24]. India, a close neighboring of Nepal, has also reported a high number of histoplasmosis cases in West Bengal and Assam till 2018[24]. From, 1995 to 2018, about 388 cases of $H$. capsulatum has been reported, and the number is in increasing order in India [24]. In recent years, an increasing number of histoplasmosis cases have been recognized among HIV-positive and/or diabetic patients in India [1]. In case of Nepal, histoplasmosis has only been described four times, Amatya et al., (2014) reported that the 
cases of histoplasmosis in an Indian national who traveled to Nepal for medical care and once in a Nepali citizen, making this the second case described in the literature by Subedi et al., (2016) [25]. Recently, another case has been published by Sharma and Adhikari (2019). According to them, a 52 years old man with diabetes shows adrenal involvement in histoplasmosis [26].

Additionally, one case has reported in a Nepalese migrant to the USA with evidence of infection being acquired in Nepal by Gandi et al., in 2015 [27]. Nepal and India have an open border, and a large number of Nepalese migrants is directly dependent on Indian economic and cultural aspects such as employment, education, social relationship, and trade among other elements. Such socioeconomic relationship directly has an impact on the dissemination of the diseases, including histoplasmosis.

\section{Identification of $\mathrm{H}$. Capsulatum and diagnosis of histoplasmosis}

Traditionally, identification of causative agent of histoplasmosis is carried out through tissue culture or body fluids at $25^{\circ} \mathrm{C}$ on Sabouraud's dextrose agar to allow the growth of mycelial phase of $H$. capsulatum. For the proper growth of mold, it takes about six weeks. After the incubation period, two different types of conidia are formed. The tuberculate or macroconidia are of about $8-15 \mu \mathrm{m}$ in diameter and have a thick wall. Similarly, the microconidia are tiny about $2-4 \mu \mathrm{m}$ in diameter and are more infectious when compared to the more abundant conidia. However, the procedure of culturing is timeconsuming [28].

Likewise, histopathological examination is another technique for detection of the Histoplasma. It is generally done for severely ill patients [29]. The method includes tissue biopsy. During the histopathological observation, other organisms also could mimic the appearance like that of $H$. capsulatum. So, this could be eliminated by using another stain like methenamine silver or periodic acid- Schiff stains, which visualize $H$. capsulatum [30]. Detection of circulating Histoplasma associated antigen in urine and serum is another diagnostic option to detect the presence of the fungi using an immunological technique like sandwich enzyme immunoassay (EIA), and it was first described in 1986 [31]. This technique generally uses $H$. capsulatum antigen, which is a polysaccharide, a polyclonal rabbit antiHistoplasma immunoglobulin G linked to biotin, and horseradish peroxidase $[4,18]$. The assay also shows the highest sensitivity against AIDS patients who had disseminated histoplasmosis [9].

Antibody tests are also performed to detect several forms of Histoplasma cases. There are two standard assays for antibody detection they are, (i) complement fixation (CF) test, which is based on the use of two separate antigens- yeast and mycelial (or histoplasmosis)- and (ii) immunodiffusion (ID) assay [32]. The ID assay tests generally detect the presence of $\mathrm{M}$ and $\mathrm{H}$ precipitin bands. An $M$ band often existing in persistent types of histoplasmosis and lasts for many months to years after the infection. An $\mathrm{H}$ band is also indicative of the severe form of histoplasmosis [17]. The ID assay is approximately only $80 \%$ sensitive but is more specific than CF assay because in $\mathrm{CF}$ assay, cross-reaction may occur with other fungal infections like sarcoidosis [33].

\section{Molecular identification of Histo- plasmosis}

In recent days, the molecular approach is widely used as a reliable and rapid technique for the detection of diseases with higher sensitivity and specificity. At present, the use of chemoluminescence labeled DNA probe for the detection of specific sequences of ribosomal RNA (rRNA) has been able to detect and confirm all the $H$. capsulatum isolates from the culture [32]. AccuProbe is one of them, which is based on the principle of nucleic acid hybridization. A single-stranded chemiluminescent labeled DNA probe is complementary to the sequence of ribosomal RNA of fungus. After the rRNA released from fungi, the steady DNA-RNA hybrid formed and that hybrids are detected and measured by using Hologicluminometer. A positive result is given by reading equal to or higher than the predetermined cut-off values, and an adverse 
effect is the cost less than that of cut-off values [39].

\section{Polymerase chain reaction (PCR) Assay}

PCR is based on the amplification of the fungal gene for identifying mycoses. In2003, Guedes et al. designed a nested PCR based technique for the rapid detection of fungi. The oligonucleotides used in this method is based on the $\mathrm{M}$ - antigens of $H$. capsulatum var. capsulatum. The oligonucleotidesMsp1F - Msp1R (pair 1) and $M s p 2 F-M s p 2 R$ (pair 2) results amplicon of size $111 \mathrm{bp}$ and $279 \mathrm{bp}$, respectively[34]; (Msp1F: 5' ACA AGA GAC GAC GGT AGC TTC ACG - 3' andMsp1R: 5' - GCG TTG GGG ATC AAG CGA TGA GCC - 3'), (Msp2F: 5' - CGG GCC GCG TTT AAC AGC GCC - 3' and Msp2R: 5' - ACC AGC GGC CAT AAG GAC GTC - 3'). The PCR reaction was performed with 100ng DNA amplified in a $25 \mu 1$ reaction using 20 pmol of oligonucleotides(35 cycles of denaturation, annealing, and chain extension) [34].

By using this protocol, $100 \%$ specificity and accuracy were mentioned in the 31 examined strains from the animal, soil, and human [34]. The results also confirmed the absence of other fungal strains such as $H$. capsulatum var. farciminosum, Paracoccidioides brasiliensis, Candida spp., Sporothrix schenckii, Cryptococcus neoformans,etc in the amplified products[34].

\section{Real-time PCR (RT - PCR)}

The real-time RT-PCR or qPCR shows promising results in the diagnosis of histoplasmosis infection, which currently identified the H. capsulatum among several other fungi [35]. Simon et al., (2010) carried out research for the detection of $H$. capsulatum based on real-time PCR using the TaqMan probe [36]. For this study, specific primers and probe (TaqManlabeled with fluorescent dye 6carboxyfluorescein at $5^{\prime}$ end and a nonfluorescent quencher at the $3^{\prime}$ end) were used for the analysis of the internal transcribed spacer (ITS) region of the rRNA. The oligonucleotides HcITS-167F (5'AACGATTGGCGTCTGAGCAT-3') and HcITS229R (5'-GAGATCCGTTGTTGAAAGTTTTGA$\left.3^{\prime}\right)$ were used. The following probeHcITS-188P
5'-6- FAM- AGAGCGATAATAATCC- MGB -3') was used. The specificity of RT- PCR was calculated to be $95.4 \%$ in comparison to the culture method. During the process, there is no PCR inhibitor detected. Among the 275 samples which were previously reported to be negative in culture method, 11 samples were reported as positive by RT-PCR with a specificity of $96.0 \%$. Similarly, among the 341 samples tested, zero nonspecific signals were recorded [36].

Despite this promising research result, for the detection of $H$. capsulatum in clinical samples, there are no currently FDA-approved molecular assays available. Moreover, to date, the molecular approach for the detection of histoplasmosis is in a developing stage. Similarly, an improved and reliable technique has to be designed as a dependable approach for the detection of the disease. Regarding the future accessibility of molecular techniques for the detection of histoplasmosis is not unclouded in resource-limited countries like Nepal [37].

\section{Treatment of Histoplasmosis}

Based on the current status, most patients with mild acute histoplasmosis limited to lungs will be resolved after a month without specific treatment. However, the disseminated or the severe form of histoplasmosis should be treated with antifungal agents[17].

The treatment of chronic and severe form of pulmonary histoplasmosis is generally done by prescribing amphotericin B $(3.0-5.0 \mathrm{mg} / \mathrm{kg}$ daily for 1-2 weeks, intravenously), followed by itraconazole ( $200 \mathrm{mg} 3$ times per day for 3 days and after that $200 \mathrm{mg}$ for two times a day, for a total of 3 months) is required and recommended[38]. Similarly, for progressive disseminated histoplasmosis amphotericin B $(3.0 \mathrm{mg} / \mathrm{kg}$ daily) is recommended for about 715days, which is followed by oral drug itraconazole having $200 \mathrm{mg}$ concentration for daily three times for three days and after that $200 \mathrm{mg}$ daily for two times for a total of at least a year). Likewise, for the patients with the immunosuppressed condition, itraconazole daily $(200 \mathrm{mg})$ is recommended as prophylaxis[19,38]. 


\section{Research status of histoplasmosis in Nepal}

In Nepal, there is poor hygienic practices and sanitation in major parts of the country and is offering the home for the continuous emergence and re-emergence of several life-threatening infectious diseases. So, Histoplasmosis could be a serious issue of public health in days to come. In Nepal, the majority of the population lives in rural areas with minimal health care facilities. The burden of the disease is much higher in rural settings compared to the urban areas. Although the pattern of diseases might change regularly, infectious diseases remain the leading cause of mortality and morbidity in Nepal. In many rural settings, cases of some diseases are often identified only through their clinical signs and symptoms. Lack of proper public health awareness and treatment of infectious diseases made people prone to several fungal infections, including Histoplasmosis, which may be a severe issue [40]. As the pervasiveness of HIV/AIDS and Tuberculosis are budding swiftly, the infection from the Histoplasma may lead to a significant effect [41]. Thus, careful diagnosis and treatment on time are paramount to control the future outbreaks of histoplasmosis. Moreover, the probability of undiagnosed histoplasmosis dissemination also urges for the need of proper diagnostic strategies of Histoplasma spp. infection in Nepal. As per institutional information, there is no routine examination and treatment of histoplasmosis infection in Tribhuvan UniversityTeaching Hospital and National Public Health Laboratory (NPHL-Nepal), while both of them are considered as reference health service centres of the country.

\section{Challenges in Nepal}

As Nepal is under the way of development, the budgeting for the health and its related sector is very less. Lack of proper investment in the health sector for the diagnosis and treatment of diseases is a significant problem in Nepal. Death of hundreds of people by unknown disease is also prevalent. Lack of proper government strategies and operative ventures in the health sector has been a cardinal cause of those strange deaths. Allocation of the budget on the health sector is only attentive to the treatment of some severe disease, which shows that the country has less interest in disease diagnosis. It is one of the significant consequences of the death of people from unknown conditions. Therefore, early diagnosis of histoplasmosis is mandatory to reduce the burden of illness.

\section{Conclusion}

The clear and accurate picture of the dissemination of histoplasmosis is complicated to understand in the resource-limited clinical sector of Nepal. Many factors could contribute to this, like lack of proper lab facilities, lack of expertise on fungal infections, etc. Additionally, not much reliable data are available from the government and academia due to incompetence in the diagnosis strategies. The published data are also in the form of case reports based on a hospital visit. It is already emphasized that the pockets of endemicity do exist in Nepal, which could lead to a significant impact on public health. Hence, careful considerations and the molecular approach of disease identification may be a suitable alternative. To address this problem, further research should be done immediately in Nepal

\section{Conflict of Interest}

The authors declare no conflict of interest.

\section{References}

1. Chakrabarti A, Slavin MA :Endemic fungal infections in the Asia-Pacific region. Med Mycol 2011 49: 337-344.

2. Stobierski MG, Hospedales CJ, Hall WN, Robinson-Dunn B, Hoch D, Sheill DA: Outbreak of histoplasmosis among employees in a paper factory - Michigan. J Clin Microbiol 1993 34:1220-1223.

3. Gajurel K, Dhakal R, Deresinski S: Histoplasmosis in transplant recipients. Clinical Transplantatfile 2017 13:1-7.

4. Wheat LJ, Azar MM, Bahr NC, Spec A: Histoplasmosis. Chest. 2016 30: 207-227.

5. Kilburn CD, McKinsey D S: Recurrent massive pleural effusion due to pleural, pericardial, and epicardial fibrosis in histoplasmosis. Chest. 1991 100: 1715-1717.

6. Holbrook ED, Kemski MM, Richer SM, Wheat LJ, Rappleye CA: Glycosylation and immunoreactivity of the Histoplasma capsulatum Cfp4 yeast-phase exoantigen. 
Infect Immun. 2014 82:4414-4425.

7. Assi M, Martin S, Wheat LJ, Hage C, Freifeld A, Avery R, et al.: Histoplasmosis after solid organ transplant. Clin Infect Dis. 2013 57:1542-1549.

8. Kauffman CA, Israel KS, Smith JW, White AC, Schwarz J, Brooks GF: Histoplasmosis in immunosuppressed patients. Am J Med. 1978 64: 923-932.

9. Guimarães AJ, Nosanch JD, Zancopé-Oliveira RM: Diagnosis of histoplasmosis. Brazilian J Microbiol. 2006 37:1-13.

10. Gugnani HC: Histoplasmosis in Africa: a review. Indian J Chest Dis Allied Sci. 2000 42: 271-277.

11. Faiolla RCL, Coelho MC, Santana RdeC, Martinez R: Histoplasmosis in immunocompetent individuals living in an endemic area in the Brazilian Southeast. Rev Soc Bras Med Trop. 2013 46: 461-465.

12. Eissenberg LG, Goldman WE: Histoplasma variation and adaptive strategies for parasitism: New perspectives on histoplasmosis. Clin Microbiol Rev. 1991 4: 411-421.

13. Pervez MM, Cobb B, Matin N, Shahrin L, Ford ER, Pietroni M: Disseminated histoplasmosis in a patient with advanced HIV disease-lessons learnt from Bangladesh. J Heal Popul Nutr. 2010 28:305307.

14. Guha A, Kulkarni HS: Histoplasmosis Patient Information Series. Am J Respir Crit Care Med. 2012 185: 1-2.

15. Silverman FN, Schwarz J, Lahey E: Histoplasmosis. Am J Med 1945 6: 410-460.

16. De D, Nath UK: Disseminated Histoplasmosis in Immunocompetent Individuals- not a so Rare Entity, in India. Med J of Hem and Infe Dis. 2015 1: 3-7.

17. Kauffman CA: Histoplasmosis: A clinical and laboratory update. Clin Microbiol Rev. 2006 19: 110-122.

18. Kauffman CA: Histoplasmosis: A clinical and laboratory update. Clin Microbiol Rev. 2007 20:115-132.

19. Bahr NC, Antinori S, Wheat LJ, Sarosi G A: Histoplasmosis Infections Worldwide: Thinking Outside of the Ohio River Valley. Curr Trop Med Reports. 2015 2:70-80.

20. Thapa S, Jha SC, Trotter AB: Images in clinical tropical medicine persistent fever and skin lesions due to histoplasmosis in a boy from rural Nepal. Am J Trop Med Hyg. 2016 94: 70-80.

21. Karki KB, Maskey J, Giri M, Pandey AR, Makai P, Subedi R, et al: Assessment of Chronic Kidney Disease Support Program of Government of Nepal. Nepal Health Research Council, Nepal 2016.

22. Acharya RP: Epidemic of Diabetes in Urban Nepal - Time To Act. J Nepal Med Assoc. 2003
42:1-2.

23. NCASC: Fact sheet 1: HIV Epidemic Update of Nepal Facts about HIV Epidemic in Nepal HIV Estimates in Nepal. 2018.

24. Randhawa HS, Gugnani HC: Occurrence of Histoplasmosis in the Indian SubContinent: An Overview and Update. J Med Res Pr. 2018 7: 3-71.

25. Amatya R, Koirala R, Khanal B, Gurung R, Rijal A, Dhakal K: Case Report histoplasmosis : first case from nepal, 2010 Department of Microbiology. Nepal 60-63.

26. Sharma N, Adhikari R: Adrenal involvement in histoplasmosis. J Pathol Nep. 2019 9: 15021504.

27. Gandhi V, Singh A, Woods GL, Epelbaum O: A 66-year-old woman with fever, cough, and a tongue lesion, Chest. 2015 147: 140-147.

28. Mansoor CA, Bhargavan PV, Rajanish R, and Nair LR: Disseminated histoplasmosis. Indian J Orthop. 2013 47: 639-642.

29. Hage CA, Connolly P, Horan D, Durkin M, Smedema $M$, Zarnowski $R$, et al: Investigation of the efficacy of micafungin in the treatment of histoplasmosis using two North American strains of Histoplasma capsulatum. Antimicrob Agents Chemother. 2011 55: 4447-4450.

30. Gudala M: Delayed Diagnosis of Pulmonary Histoplasmosis in an Immunocompetent Young Asthmatic Female from Florida, a Non-Endemic Area for Histoplasma Capsulatum. Med Mycol Open Access. 2015 1:1-6.

31. Rajeshwari M, Xess I, Sharma MC, Jain D: Acid-fastness of Histoplasma in surgical pathology practice. J Pathol Transl Med. 2017 51, 482-487.

32. Azar MM, Hage CA: Laboratory diagnostics for histoplasmosis. J Clin Microbio. 2017 55: 1612-1620.

33. Picardi JL, Kauffman CA, Schwarz J, Phair JP: Detection of precipitating antibodies to Histoplasma capsulatum by counterimmunoelectrophoresis. Am Rev Respir Dis. 1976 114: 171-176.

34. Guedes HL, Guimarães AJ, Muniz Mde M, Pizzini CV, Hamilton AJ, Peralta JM et al: PCR assay for identification of Histoplasma capsulatum based on the nucleotide sequence of the $\mathbf{M}$ antigen. J Clin Microbiol. 2003 41(2): 535-539.

35. Muraosa Y, Toyotome T, Yahiro M, Watanabe A, Shikanai-Yasuda MA, Kamei K: Detection of Histoplasma capsulatum from clinical specimens by cycling probe-based real-time PCR and nested real-time PCR. Med Mycol. 2016 54: 433-438.

36. Simon S, Veron V, Boukhari R, Blanchet D, and Aznar C: Detection of Histoplasma capsulatum DNA in human samples by realtime polymerase chain reaction. Diagn 
Microbiol Infect Dis. 2010 66: 268-273.

37. Ha HTT, Ohnoc H, Tram NT, My TN, Hai PT, Hoa LM, et al: Histoplasmosis in Pulmonary Infection Patients from Hospitals in Hanoi, Vietnam. Appl Microbiol. 2017 3: 3-5.

38. Wheat LJ, Freifeld AG, Kleiman MB, Baddley JW, McKinsey DS, Loyd JE et al: Clinical Practice Guidelines for the Management of Patients with Histoplasmosis: 2007 Update by the Infectious Diseases Society of America. Clin Infect Dis. 2007 45: 807-825.

39. Wu XS, Yang YY, Zhang LJ, Lu GM: Diagnosis of Histoplasmosis. Chinese J Radiol. 2008 42: 771-773.

40. Baker J, Setianingrum F, Wahyuningsih R, Denning DW: Mapping histoplasmosis in South East Asia - implications for diagnosis in AIDS. Emerg Microbes Infect. 2019 8:11391145.

41. Nacher $M$, Blanchet $D$, Bongomin $F$, Chakrabarti A, Couppié P, Demar M, et al: Histoplasma capsulatum Antigen Detection Tests As An Essential Diagnostic Tool For Patients With Advanced HIV Disease In Low And Middle Income Countries: A Systematic Review Of Diagnostic Accuracy Studies. PLoS Negl Trop Dis. 2018 12:1-12. 